\title{
A virus-like particle vaccine for epidemic Chikungunya virus protects nonhuman primates against infection
}

\author{
Wataru Akahata ${ }^{1}$, Zhi-Yong Yang ${ }^{1}$, Hanne Andersen ${ }^{2}$, Siyang Sun ${ }^{3}$, Heather A Holdaway ${ }^{3}$, Wing-Pui Kong ${ }^{1}$, \\ Mark G Lewis ${ }^{2}$, Stephen Higgs ${ }^{4}$, Michael G Rossmann ${ }^{3}$, Srinivas Rao ${ }^{1} \&$ Gary J Nabel $^{1}$
}

\section{Chikungunya virus (CHIKV) has infected millions of people} in Africa, Europe and Asia ${ }^{1,2}$ since this alphavirus reemerged from Kenya in 2004. The severity of the disease and the spread of this epidemic virus present a serious public health threat in the absence of vaccines or antiviral therapies. Here, we describe a new vaccine that protects against CHIKV infection of nonhuman primates. We show that selective expression of viral structural proteins gives rise to virus-like particles (VLPs) in vitro that resemble replication-competent alphaviruses. Immunization with these VLPs elicited neutralizing antibodies against envelope proteins from alternative $\mathrm{CHIKV}$ strains. Monkeys immunized with VLPs produced hightiter neutralizing antibodies that protected against viremia after high-dose challenge. We transferred these antibodies into immunodeficient mice, where they protected against subsequent lethal CHIKV challenge, indicating a humoral mechanism of protection. Immunization with alphavirus VLP vaccines represents a strategy to contain the spread of CHIKV and related pathogenic viruses in humans.

CHIKV, a mosquito-borne alphavirus in the family Togaviridae, was first isolated in Tanzania in 1952 (ref. 3). Infection by this virus in humans is characterized by rash, high fever and, its hallmark feature, severe arthritis that can persist for years ${ }^{1,2}$. An epidemic strain of CHIKV appeared on Reunion Island in 2005 and has since spread to more than 18 countries $^{4}$ (see also http://www.cdc.gov/ncidod/dvbid/chikungunya/ CH_GlobalMap.html). The dissemination of this epidemic virus is associated with genetic mutations that facilitated its adaptation to a new insect vector, the Asian tiger mosquito, Aedes albopictus ${ }^{5}$, that survives in temperate climates and is widely distributed ${ }^{6}$. CHIKV continues to cause substantial morbidity resulting in considerable economic losses, and vaccine development remains a high priority.

CHIKV is composed of a positive, single-stranded genomic RNA of 12,000 nucleotides encoding four nonstructural and five structural polyproteins ${ }^{7}$. The nonstructural proteins, nsP1, nsP2, nsP3 and nsP4, are required for virus replication; the structural proteins, which consist of capsid and envelope proteins (E1, E2, E3 and 6K), are synthesized as polyproteins and are cleaved by capsid autoproteinase and signalases ${ }^{7}$
(Fig. 1a). Given the similarity of the CHIKV genomic structure to those of other alphaviruses, CHIKV is expected to encode spikes on the virion surface that are each formed by three E1-E2 heterodimers ${ }^{8,9}$, where the E1 glycoproteins mediate fusion and the E2 glycoproteins interact with the host receptor ${ }^{7}$. Phylogenetic analysis of CHIKV has revealed three genotypes: Asian, East, Central and South African and West African. All strains are highly related, with $95.2-99.8 \%$ amino acid similarity ${ }^{10}$. Although the CHIKV strains vary, the individual strains are antigenically related, so it is potentially possible to design a vaccine that works against heterologous strains ${ }^{11}$.

We first analyzed the immunogenicity and cross-reactivity of two disparate strains, 37997 from the older West Africa lineage and LR2006 OPY-1, the more recent strain responsible for the current epidemic. To examine the specificity of viral entry into target cells and the sensitivity of these two strains to neutralizing antibodies, we developed lentiviral vector reporters pseudotyped with glycoproteins from these two CHIKV strains. Such vectors allow analysis of the mechanism and the specificity of entry, and help to quantify antibody neutralization more readily than wild-type viruses that pose considerable biohazard concerns for routine laboratory analyses. They therefore provide a useful tool for the study of CHIKV entry as well as for vaccine development ${ }^{12-14}$. We inserted CHIKV envelope genes encoding the native polypeptide, E3-E2-6K-E1 (Fig. 1a), from the 37997 and LR2006 OPY-1 strains into an expression vector $\left(\mathrm{E}_{37997}\right.$ and $\left.\mathrm{E}_{\mathrm{OPY}-1}\right)$. We verified incorporation of each set of CHIKV envelope proteins into the pseudotyped lentiviral vectors by buoyant density gradient sedimentation of the virus. We detected CHIKV envelope and HIV-1 Gag proteins in the same buoyant density fraction as lentivirus particles (Supplementary Fig. 1). The 37997 and LR2006 OPY-1 CHIKV-pseudotyped lentiviral vectors infected several cell lines permissive for viral replication ${ }^{15}$ (Fig. 1b). We then measured their ability to express a luciferase reporter in 293A human renal epithelial cells and found that entry was dose dependent (Fig. 1b), whereas an empty control vector, devoid of CHIKV envelope proteins, did not infect these cell lines. To determine whether entry occurred through the same mechanism used by native virus, we analyzed $\mathrm{pH}$ and the endosome dependence of entry ${ }^{13}$. CHIKV infects cells through $\mathrm{pH}$-dependent cell fusion ${ }^{15}$; thus, addition of ammonium chloride or chloroquine, which prevent acidification of the endosome, caused a dose-dependent reduction

${ }^{1}$ Vaccine Research Center, National Institute of Allergy and Infectious Diseases, US National Institutes of Health, Bethesda, Maryland, USA. ${ }^{2}$ Bioqual, Inc., Rockville, Maryland, USA. ${ }^{3}$ Department of Biological Sciences, Purdue University, West Lafayette, Indiana, USA. ${ }^{4}$ Department of Pathology, University of Texas Medical Branch, Galveston, Texas, USA. Correspondence should be addressed to G.J.N. (gnabel@nih.gov). 
a

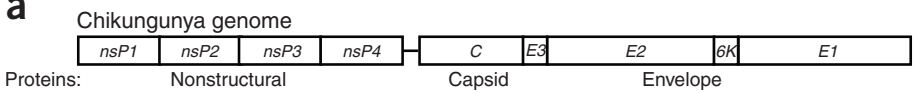

b

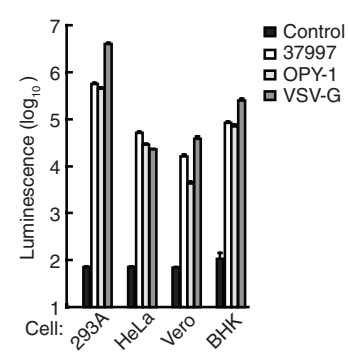

C
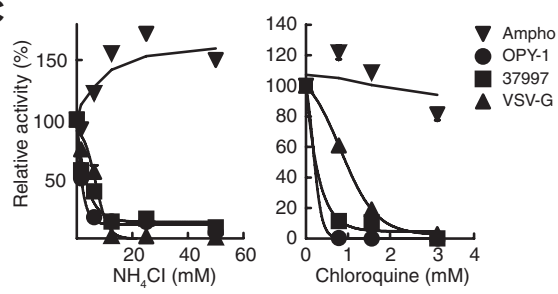

d

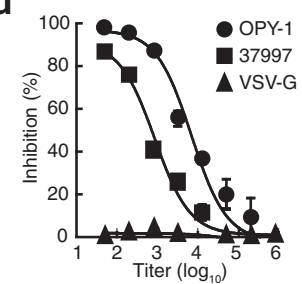

Figure 1 Characterization of CHIKV E-pseudotyped lentiviral vectors. (a) Schematic representation of the CHIKV genome and CHIKV E expression vector used for incorporation of CHIKV E from strains 37997 and LR2006 OPY-1 into pseudotyped lentiviral vectors. The CHIKV genome consists of the following genes: nonstructural polyproteins $n s P 1, n s P 2, n s P 3$ and $n s P 4$ and structural polyproteins capsid $(C)$ and envelope (E: E3, E2, 6K and E1) (top). The polypeptide $E$ genes from strains 37997 and LR2006 OPY-1 were inserted into an expression vector (bottom; a schematic representation of the vector is in Supplementary Fig. 1a). (b) Infectivity of the indicated pseudotyped lentiviral vectors in several CHIKV-permissive cell lines including 293A human renal epithelial, HeLa cervical epithelial, Vero renal epithelial and baby hamster kidney (BHK) cells. The pseudotyped vectors were standardized by HIV-1 Gag p24 (left) or the indicated concentration of p24 and used to infect 293A cells (right). After incubation with pseudotyped vectors for $24 \mathrm{~h}$, cells were lysed and luciferase activity was measured. Error bars show the s.e.m. (c) pH-dependent entry of CHIKV pseudotyped lentiviral vectors incubated in the presence of the indicated amounts of ammonium chloride (left) and chloroquine (right). Data are presented as the percentage of activity at the indicated dose relative to activity with no treatment. Error bars show the s.e.m. (d) Neutralization measured with pseudotyped lentiviral vectors in sera from immune mice infected with CHIKV strain S-27. Sera were incubated at the indicated dilutions with VSV-G-, CHIKV strain 37997- or LR2006 OPY-1 E-pseudotyped lentiviral vectors, and the mixture was used to infected 293 A cells. No inhibition was observed with control nonimmune antisera. Error bars show the s.e.m. and in many cases are too small to be visible.

in CHIKV pseudotyped vector entry (Fig. 1c). We observed a similar inhibition of entry with a lentiviral vector pseudotyped with vesicular stomatitis virus G protein (VSV-G), which is known to mediate entry in this fashion, but not with amphotropic murine leukemia virus glycoprotein 70 , which mediates entry by a $\mathrm{pH}$-independent mechanism ${ }^{13,16}$. These findings indicate that lentiviral vectors pseudotyped with CHIKV envelope mediate entry through the same mechanism as wild-type virus. Next, we examined a commercially available immune serum from mice infected with an established CHIKV strain. Incubation of the immune sera with the CHIKV-pseudotyped lentiviral vector, but not a VSV-Gpseudotyped vector, inhibited entry (Fig. 1d). We could therefore quantify the specificity and potency of the neutralizing antibodies without needing to expose laboratory workers to infectious virus.

We next analyzed eukaryotic expression vectors encoding C-E3-E26K-E1 from strains 37997 and LR2006 OPY-1 (C- $\mathrm{E}_{37997}$ and C-E $\left.\mathrm{E}_{\mathrm{OPY}-1}\right)$ for their ability to give rise to VLPs. We transfected 293T human kidney cells with C- $\mathrm{E}_{37997}$ or C-E $\mathrm{E}_{\mathrm{OPY}-1}$ or $\mathrm{E}_{37997}$ or $\mathrm{E}_{\mathrm{OPY}-1}$ (Fig. 2a) and confirmed expression in the cell lysates by western blotting (Fig. 2a).

Figure 2 Characterization of CHIKV VLPs. (a) Top, schematic representation of the CHIKV C-E or E expression vectors used for DNA vaccine and VLP production. The $\mathrm{CHIKV}$ structural polyproteins capsid plus envelope (C-E) or E alone from strains 37997 and LR2006 OPY-1 were inserted into a CMV/R expression vector ${ }^{14}$. Bottom, western blotting of 293T cells transfected with the above plasmids $48 \mathrm{~h}$ after transfection ${ }^{33}$, as previously described (Supplementary Methods). (b) Left, buoyant density fractionation of VLPS purified from fractionated supernatants of $293 \mathrm{~F}$ cells transfected with the $\mathrm{C}$-E expression vector ( $\mathrm{C}_{-\mathrm{E}_{37997}}$ (top) and protein expression (bottom), as determined by western blot analysis. Right, transmission electron microscopic image of fractionated VLPs. Scale bar, $100 \mathrm{~nm}$. (c) Cryoelectron microscopy reconstruction of CHIKV VLP. Left, the central cross-section through the cryoelectron microscopy maps of CHIKV VLP. The orientations of the icosahedral (two-, three- and fivefold) axes as well as the quasi-threefold (q3) axis are shown with white lines. Right, a shaded-surface representation of the three-dimensional density map of CHIKV VLP, viewed along an icosahedral twofold axis, resembling Sindbis virus ${ }^{8,9,18}$ (Supplementary Fig. 2). The white triangle marks the boundary of an icosahedral asymmetric unit. The numbers show the positions of the icosahedral two-, three- and fivefold axes limiting an asymmetric unit. The map is calculated to $18 \AA$ resolution.

We also detected capsid and E1 and E2 proteins in the supernatant by western blotting after transfection of the $\mathrm{C}-\mathrm{E}_{37997}$ or $\mathrm{C}-\mathrm{E}_{\mathrm{OPY}-1}$ vector, suggesting that CHIKV VLPs had been generated. We purified VLPs by

\section{a} \begin{tabular}{ll|l|l|l|l|l|}
\hline CMV & $R$ \\
\hline
\end{tabular}
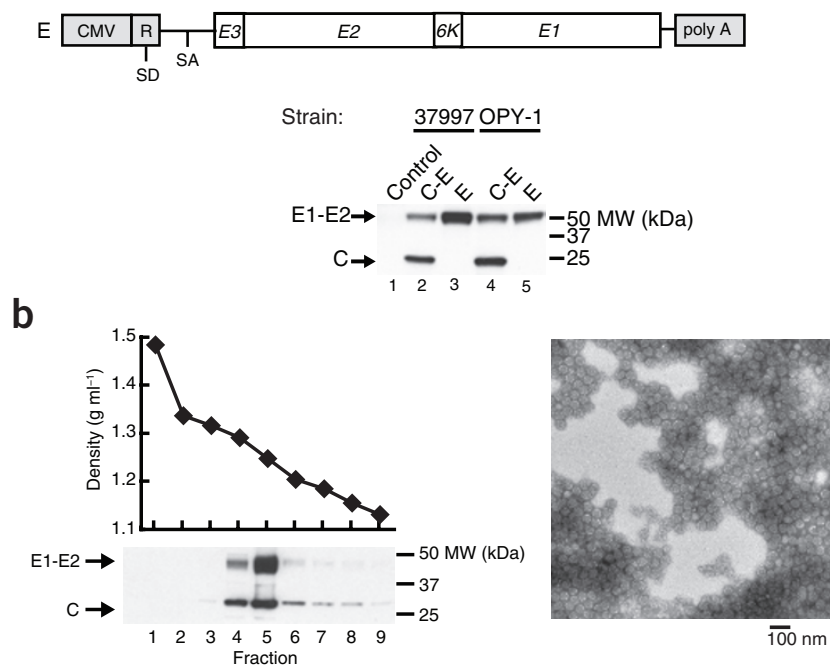

C

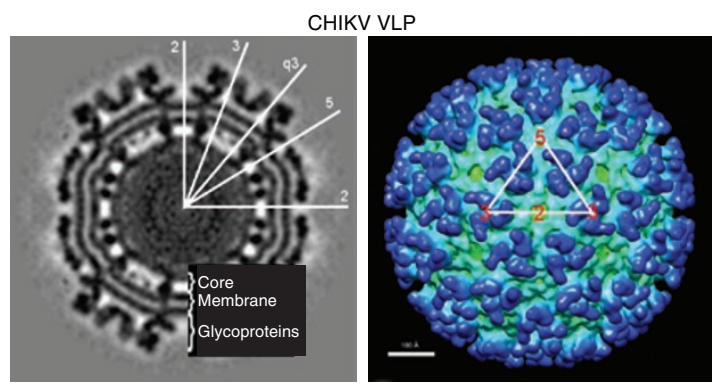




\section{LETTERS}

a

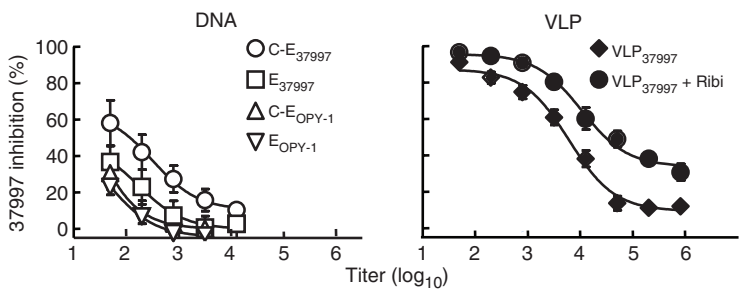

C

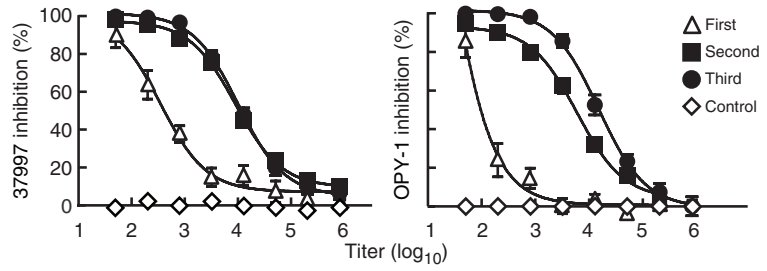

b

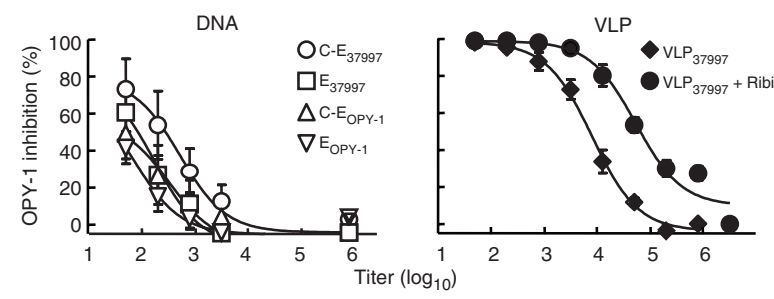

d

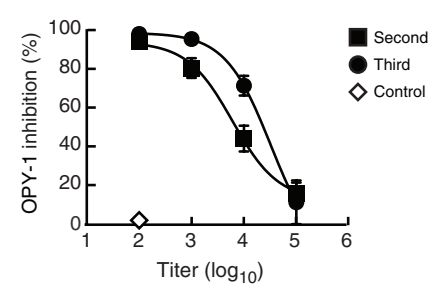

Figure 3 Neutralization of CHIKV strains 37997 and LR2006 OPY-1 after DNA or VLP vaccination in mice and monkeys. (a,b) Neutralization titers of sera from immunized BALB/c mice measured with CHIKV strain 37997 (a) or LR2006 OPY-1 (b) E-pseudotyped lentiviral vectors. Mice were immunized intramuscularly three times with the indicated DNA or VLP $37997 . V \mathrm{VP}_{37997}$ with or without Ribi adjuvant was injected twice. The experiment was performed in triplicate. The symbols show the average of the five mice, and error bars show the s.e.m. The curve fit was calculated by Prism software. (c) Neutralization titers of sera from immunized monkeys were determined with CHIKV strain 37997 (left) or LR2006 OPY-1 (right) E-pseudotyped lentiviral vectors. Rhesus monkeys were immunized with VLP 37997 or PBS (control) intramuscularly three times, and sera were collected $10 \mathrm{~d}$ later. The symbols show the average of the six monkeys, and error bars show the s.e.m. (d) Neutralizing activity against CHIKV LR2006 OPY-1 in immunized monkeys' sera after the second and third immunizations, as confirmed by a standard PRNT. The symbols show the average of the six monkeys, and error bars show the s.e.m.

buoyant density gradient sedimentation. The yield of VLPs from strain 37997 was $10-20 \mathrm{mg} \mathrm{l}^{-1}$, approximately 100 times more than that from strain LR2006 OPY-1. For this reason and because of the high degree of amino acid similarity among CHIKV strains, we used the 37997 strain to produce VLPs. Fractionation of clarified supernatant showed the greatest incorporation of E1 complexed with E2 (E1-E2) into the VLPs at a density of $1.2 \mathrm{~g} \mathrm{ml}^{-1}$ (Fig. 2b), comparable to the density of wild-type $\mathrm{CHIKV}^{17}$. Examination of the purified fraction from strain 37997 by electron microscopy revealed VLPs with the same morphologic appearance as wild-type virus (Fig. 2b).

Cryoelectron microscopy and three-dimensional image reconstruction, assuming icosahedral symmetry, showed that the VLPs had an external diameter of $65 \mathrm{~nm}$ and a core diameter of $40 \mathrm{~nm}$ (Fig. 2c). The potent immunogenic E1-E2 glycoproteins are organized into 240 heterodimers, assembled into 80 glycoprotein spikes arranged with triangulation number $(T)=4$ quasisymmetry on the surface of the VLPs (Fig. 2c), closely similar to the structure of Sindbis virus (Supplementary

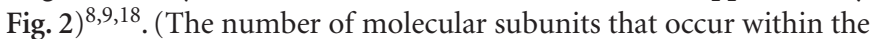
icosahedral asymmetric unit with quasisimilar environments is known as the triangulation number, $T$, and is a crucial determinant for virus assembly ${ }^{19}$.)

Because of the ease of DNA vaccine preparation and its broad applicability, we compared the relative immunogenicity of plasmid DNA to VLP vaccines in mice by immunizing with DNA vaccines encoding C-E or E (strains 37997 and LR2006 OPY-1) or VLPs from strain 37997 $\left(\mathrm{VLP}_{37997}\right)$ in the presence or absence of the Ribi immune stimulatory adjuvant. BALB/c mice injected twice intramuscularly with VLPs with adjuvant generated the highest-titer neutralizing responses against both the homologous strain 37997 (Fig. 3a; half-maximal inhibitory concentration $\left(\mathrm{IC}_{50}\right)$ titer of 10,703) and the heterologous strain LR2006 OPY-1 (Fig. 3b; IC $_{50}$ titer of 54,600). Although three immunizations with the plasmids encoding $\mathrm{C}$-E and $\mathrm{E}$ from both strains elicited neutralizing responses, these titers were less than $5 \%$ of those elicited in VLPimmunized mice (Fig. 3a,b; $\mathrm{IC}_{50}$ titer of 319 for DNA C-E $\mathrm{E}_{37997}$ against strain 37997; 525 for DNA C-E 37997 against strain LR2006 OPY-1). These results suggested that the VLPs elicit a more potent neutralizing antibody response than DNA vaccines.

To characterize the VLP-induced immune responses in a model with stronger predictive value for humans, we immunized rhesus macaques with VLPs. Monkeys received intramuscular injections of VLP 37997 or PBS alone as a control. We tested sera from immunized and control monkeys against CHIKV strain 37997-and LR2006 OPY-1-pseudotyped lentiviral vectors in vitro. All nonhuman primates immunized with VLPs developed substantial neutralizing activity to both homologous and heterologous strains after primary immunization that increased after boosting (Fig. 3c; $\mathrm{IC}_{50}$ titer of 10,219 against strain $37997, \mathrm{IC}_{50}$ titer of 15,072 against strain LR2006 OPY-1). Notably, there was slightly better neutralization of LR2006 OPY-1 compared to 37997 in both mice and monkeys, although these effects were not substantial, suggesting that the LR2006 OPY-1 virus may present a conserved epitope to the immune system better than the 37997 virus. To confirm that these antibodies neutralize CHIKV-pseudotyped lentiviral vectors similarly to wild-type infectious virus, we performed a plaque reduction neutralization test (PRNT) against the CHIKV strain LR2006 OPY-1. The antisera from the immunized monkeys mediated neutralization of LR2006 OPY-1 at a half-maximal PRNT of 40,133 (Fig. 3d). These data suggest that measurement of neutralizing antibodies with the pseudotyped lentiviral vector assay correlates with the PRNT assay of wild-type virus and that all VLP-immunized monkeys generated potent neutralizing antibody responses against $\mathrm{CHIKV}$.

To establish a CHIKV challenge model in monkeys, we injected two naive rhesus monkeys intravenously with a high-titer LR2006 OPY-1 virus stock. Viremia commenced at $6 \mathrm{~h}$ and lasted until at least $72 \mathrm{~h}$ after challenge in these monkeys, with the peak viremia at $24 \mathrm{~h}$ after infection (Supplementary Fig. 3a). As in humans, infection in nonhuman primates resulted in viremia and was not lethal. In addition, we observed a similar transient acute lymphopenia and a proinflammatory response, as measured by transient neutrophilia and an increase in monocyte counts 
a
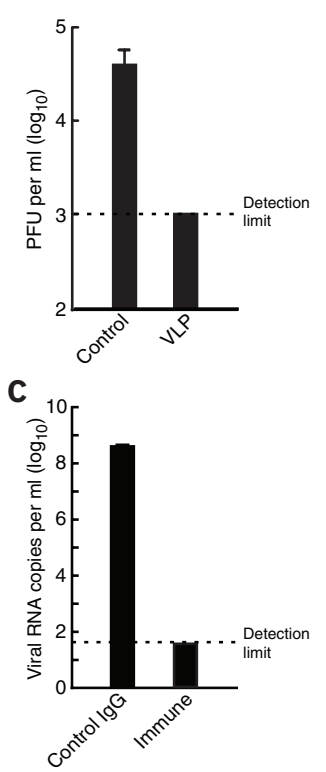

b

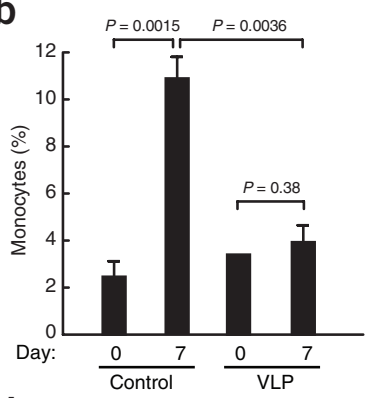

d

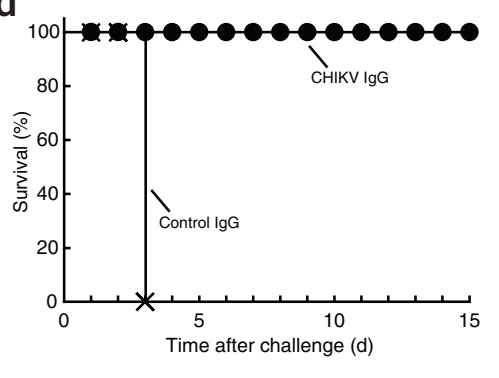

Figure 4 Protection against CHIKV LR2006 OPY-1 challenge in monkeys immunized with VLPs and in a CHIKV mouse model after passive transfer of purified IgG. (a) The peak viremia, $24 \mathrm{~h}$ after challenge, as measured by a plaque assay. Monkeys injected with PBS (Control) or immunized with $V P_{37997}(V L P)$ were challenged intravenously with $1 \times 10^{10}$ plaque-forming units (PFU) of the CHIKV strain LR2006 OPY-1. The detection limit was 1,000 PFU ml-1. Error bars represent the s.e.m. (b) The percentage of monocytes in the monkeys' white blood cells, as measured with a hematology analyzer before and $7 \mathrm{~d}$ after challenge with CHIKV. Error bars represent the s.e.m. An unpaired two-tailed $t$ test was used for statistical analysis (Control at day 0 versus day $7, P=0.0015$; VLPs at day 0 versus day $7, P=0.38$; Control versus VLPs at day $7, P=0.0036$ ). (c) The peak viremia in the mice that received IgG after challenge, as measured by quantitative RT-PCR. Purified IgG from a monkey immunized with VLPs (Immune) or a control monkey (Control lgG) was passively transferred into Ifnar $\mathrm{I}^{-/-}$mice intravenously (2 $\mathrm{mg}$ of total IgG per mouse). Recipient mice were challenged $24 \mathrm{~h}$ after IgG transfer with a lethal LR2006 OPY-1 challenge. The detection limit was 40 RNA copies per milliliter. Error bars represent the s.e.m. (d) Survival curve of mice lethally challenged with LR2006 OPY-1 after passive transfer with control IgG or IgG generated by CHIKV VLP immunization.

(Supplementary Fig. 3b). We assessed the ability of the VLP vaccine to protect against infection by intravenous challenge of monkeys immunized with VLPs or sham-immunized controls using the same high-titer LR2006 OPY-1 virus stock 15 weeks after the final immunization. In this model, all immunized monkeys controlled the challenge virus completely (Fig. 4a). In contrast, we observed viremia in all of the control monkeys injected with CHIKV, as in the pilot experiment. Similarly, monocyte counts were markedly increased in control monkeys $4 \mathrm{~d}$ after challenge, whereas they remained unchanged in vaccinated monkeys (Fig. $4 \mathrm{~b}$; control at day 0 versus day 7, $P=0.0015$; VLPs at day 0 versus day $7, P=0.38$ ). These data suggest that immunization with VLPs protects against both viremia and the inflammatory consequences of infection.

To define the mechanism of protection in these monkeys, we investigated whether or not immune IgG could protect against lethal challenge in an adoptive transfer model. Previous studies have shown that immunodeficient mice with defective type 1 interferon signaling (interferon$\alpha / \beta$ receptor-1 knockout, Ifnar $1^{-/}$) are susceptible to lethal CHIKV infection, showing pathological manifestations of infection ${ }^{20}$ and providing a model to evaluate immune mechanisms of protection. For example, it was previously shown that antibodies have a key role in pro-

tecting against CHIKV infection by passive transfer of IgG from infected humans in this immunodeficient model ${ }^{21}$. Here we intravenously transferred purified total IgG from immunized or control monkeys into these Ifnar $1^{-/}$immunodeficient mice and challenged the recipient mice intradermally with a lethal dose of LR2006 OPY-1 24 h later. Recipients of purified IgG from CHIKV-immunized monkeys showed no detectable viremia after challenge and were completely protected from lethality (Fig. 4c,d). In contrast, all mice that received purified IgG from control monkeys showed severe infection and viremia and died (Fig. 4c,d). These results indicate that humoral immune responses induced by $\mathrm{CHIKV}$ VLPs confer protection against CHIKV infection.

To date, there has been limited success in developing a safe and effective CHIKV vaccine. A live attenuated CHIKV vaccine candidate ${ }^{22,23}$ caused transient arthralgia in volunteers ${ }^{24}$. Other efforts, including a formalin-killed vaccine ${ }^{25,26}$, a Venezuelan equine encephalitis-CHIKV chimeric live attenuated vaccine ${ }^{27}$ and a consensus-based DNA vaccine $^{28}$, have not yet proven both safe and effective. The development of a safe and effective CHIKV vaccine will require additional evaluation in humans. However, the safety and efficacy of VLP vaccines in general ${ }^{29}$ make them promising candidates for further study.

VLPs are known to be highly immunogenic and elicit higher-titer neutralizing antibody responses than subunit vaccines based on individual proteins ${ }^{29-31}$. Such VLPs authentically present viral spikes and other surface components in a repetitive array that effectively elicits recognition by $\mathrm{B}$ cells to stimulate antibody secretion ${ }^{29,30}$. This recognition leads to B cell signaling and major histocompatibility complex class II upregulation that facilitates the generation of high-titer, specific antibodies. VLPs from other viruses, including hepatitis B virus and human papillomavirus, elicit high-titer neutralizing antibody responses that contribute to protective immunity in humans ${ }^{31,32}$. The vaccine described here represents what is to our knowledge the first use of recombinant VLPs to prevent infection by alphaviruses. At a time when there are no commercially available vaccines and with the emergence of CHIKV transmission from insect vectors, a VLP vaccine has the potential to have a considerable impact on the spread of this disease. Changes in trade, travel and global climate have aided the spread of mosquito species worldwide, which may potentially cause other alphavirus outbreaks. Our approach may prove useful for vaccine development against other pathogenic alphaviruses, including Western, Eastern and Venezuelan equine encephalitis viruses, O'nyong-nyong virus and Ross River virus.

\section{METHODS}

Methods and any associated references are available in the online version of the paper at http://www.nature.com/nm/.

Note: Supplementary information is available on the Nature Medicine website.

\section{ACKNOWLEDGMENTS}

We thank K. Nagashima for help with electron microscopy and J.D. Yoder for initiating cryoelectron microscopy reconstruction. We also thank A. Tislerics and J. Stein for help with manuscript preparation, B. Hartman for graphic arts and members of the Nabel lab for helpful discussions. We thank R. Seder and D.D. Pinschewer (Department of Pathology and Immunology, University of Geneva) for their kind gift of Ifnar ${ }^{-1-}$ mice, A. Ault, J.-P. Todd, A. Zajac, C. Chiedi (Vaccine Research Center) and D. Gordon (Bioqual) for plaque assay and processed animal blood samples, J. Greenhouse for RT-PCR assay, B.W. Finneyfrock, T. Jenkins and A. Dodson for animal sampling and care, K. Foulds, M. Donaldson and M. Roederer for monkey sample procedures and J. Lee for preparing 293F cells for VLP production. This research was supported by the Intramural Research Program of the Vaccine Research Center, National Institute of Allergy and Infectious Diseases, US National Institutes of Health.

\section{AUTHOR CONTRIBUTIONS}

W.A., Z.-Y.Y., S.H., S.R. and G.J.N. designed the research studies; W.A., Z.-Y.Y., H.A., S.S., H.A.H., W.-P.K., M.G.L. and S.R. performed the research; W.A. and 


\section{LETTERS}

Z.-Y.Y. contributed to development and generation of vectors; and W.A., Z.-Y.Y., H.A., S.S., H.A.H., W.-P.K., M.G.L., S.H., M.G.R., S.R. and G.J.N. analyzed data; and W.A., Z.-Y.Y., S.H., M.G.R., S.R. and G.J.N. wrote the paper.

\section{COMPETING INTERESTS STATEMENT}

The authors declare no competing financial interests.

Published online at http://www.nature.com/naturemedicine/.

Reprints and permissions information is available online at http://npg.nature.com/ reprintsandpermissions/.

1. Simon, F., Savini, H. \& Parola, P. Chikungunya: a paradigm of emergence and globalization of vector-borne diseases. Med. Clin. North Am. 92, 1323-1343 (2008)

2. Powers, A.M. \& Logue, C.H. Changing patterns of Chikungunya virus: re-emergence of a zoonotic arbovirus. J. Gen. Virol. 88, 2363-2377 (2007).

3. Ross, R.W. The Newala epidemic. III. The virus: isolation, pathogenic properties and relationship to the epidemic. J. Hyg. (Lond.) 54, 177-191 (1956).

4. Staples, J.E., Breiman, R.F. \& Powers, A.M. Chikungunya fever: an epidemiological review of a re-emerging infectious disease. Clin. Infect. Dis. 49, 942-948 (2009).

5. Tsetsarkin, K.A., Vanlandingham, D.L., McGee, C.E. \& Higgs, S. A single mutation in Chikungunya virus affects vector specificity and epidemic potential. PLoS Pathog. 3, e201 (2007).

6. Enserink, M. Entomology. A mosquito goes global. Science 320, 864-866 (2008).

7. Strauss, J.H. \& Strauss, E.G. The alphaviruses: gene expression, replication and evolution. Microbiol. Rev. 58, 491-562 (1994).

8. Cheng, R.H. et al. Nucleocapsid and glycoprotein organization in an enveloped virus. Cel/ 80, 621-630 (1995).

9. Zhang, W. et al. Placement of the structural proteins in Sindbis virus. J. Virol. 76 , $11645-11658$ (2002)

10. Arankalle, V.A. et al. Genetic divergence of Chikungunya viruses in India (1963-2006) with special reference to the 2005-2006 explosive epidemic. J. Gen. Virol. 88, 19671976 (2007).

11. Harrison, V.R., Binn, L.N. \& Randall, R. Comparative immunogenicities of chikungunya vaccines prepared in avian and mammalian tissues. Am. J. Trop. Med. Hyg. 16, 786-791 (1967)

12. Naldini, L., Blomer, U., Gage, F.H., Trono, D. \& Verma, I.M. Efficient transfer, integration, and sustained long-term expression of the transgene in adult rat brains injected with a lentiviral vector. Proc. Natl. Acad. Sci. USA 93, 11382-11388 (1996).

13. Yang, Z.-Y. et al. pH-dependent entry of severe acute respiratory syndrome coronavirus is mediated by the Spike glycoprotein and enhanced by dendritic cell transfer through
DC-SIGN. J. Virol. 78, 5642-5650 (2004)

14. Yang, Z.-Y. et al. Immunization by avian H5 influenza hemagglutinin mutants with altered receptor binding specificity. Science 317, 825-828 (2007).

15. Sourisseau, M. et al. Characterization of reemerging Chikungunya virus. PLoS Pathog. 3, e89 (2007)

16. McClure, M.O., Sommerfelt, M.A., Marsh, M. \& Weiss, R.A. The $\mathrm{pH}$ independence of mammalian retrovirus infection. J. Gen. Virol. 71, 767-773 (1990).

17. Eckels, K.H., Harrison, V.R. \& Hetrick, F.M. Chikungunya virus vaccine prepared by Tween-ether extraction. Appl. Microbiol. 19, 321-325 (1970).

18. Pletnev, S.V. et al. Locations of carbohydrate sites on alphavirus glycoproteins show that E1 forms an icosahedral scaffold. Cell 105, 127-136 (2001).

19. Caspar, D.L. \& Klug, A., Physical principles in the construction of regular viruses. Cold Spring Harb. Symp. Quant. Biol. 27, 1-24 (1962).

20. Couderc, T. et al. A mouse model for Chikungunya: young age and inefficient typeinterferon signaling are risk factors for severe disease. PLoS Pathog. 4, e29 (2008).

21. Couderc, T. et al. Prophylaxis and therapy for Chikungunya virus infection. J. Infect Dis. 200, 516-523 (2009).

22. Levitt, N.H. et al. Development of an attenuated strain of chikungunya virus for use in vaccine production. Vaccine 4, 157-162 (1986).

23. McClain, D.J. et al. Immunologic interference from sequential administration of live attenuated alphavirus vaccines. J. Infect. Dis. 177, 634-641 (1998).

24. Edelman, R. et al. Phase II safety and immunogenicity study of live Chikungunya virus vaccine TSI-GSD-218. Am. J. Trop. Med. Hyg. 62, 681-685 (2000).

25. Harrison, V.R., Eckels, K.H., Bartelloni, P.J. \& Hampton, C. Production and evaluation of a formalin-killed Chikungunya vaccine. J. Immunol. 107, 643-647 (1971)

26. Tiwari, M. et al. Assessment of immunogenic potential of Vero adapted formalin inactivated vaccine derived from novel ECSA genotype of Chikungunya virus. Vaccine 27 2513-2522 (2009)

27. Wang, E. et al. Chimeric alphavirus vaccine candidates for Chikungunya. Vaccine $\mathbf{2 6}$ 5030-5039 (2008).

28. Muthumani, K. et al. Immunogenicity of novel consensus-based DNA vaccines against Chikungunya virus. Vaccine 26, 5128-5134 (2008).

29. Chackerian, B. Virus-like particles: flexible platforms for vaccine development. Expert Rev. Vaccines 6, 381-390 (2007).

30. Bachmann, M.F. et al. The influence of antigen organization on B cell responsiveness Science 262, 1448-1451 (1993).

31. Noad, R. \& Roy, P. Virus-like particles as immunogens. Trends Microbiol. 11, 438-444 (2003).

32. Ludwig, C. \& Wagner, R. Virus-like particles-universal molecular toolboxes. Curr. Opin Biotechnol. 18, 537-545 (2007).

33. Akahata, W., Yang, Z.Y. \& Nabel, G.J. Comparative immunogenicity of human immunodeficiency virus particles and corresponding polypeptides in a DNA vaccine. J. Virol. 79, 626-631 (2005). 


\section{LETTERS}

\section{ONLINE METHODS}

Vector construction. We synthesized plasmids encoding the structural polyproteins C, E1, E2, E3 and 6K (strains 37997 and LR2006 OPY-1, GenBank EU224270 and EU224268, respectively) as previously described ${ }^{14}$ (GeneArt). We amplified plasmids encoding the polyproteins E3, E2, 6K and E1 by PCR using sense primer $5^{\prime}$-GCTCTAGACACCATGAGCCTCGCCCTCCCGGTCTTG- $3^{\prime}$ and antisense primer 5'-TGGATCCTCATTAGTGCCTGCTAAACGACA-3' (37997) and sense primer 5'-GCTCTAGACACCATGAGTCTTGCCATCCCAGTTATG-3' and antisense primer 5'-TGGATCCTCATTAGTGCCTGCTGAACGACA-3' (LR2006 OPY-1). We inserted XbaI and BamHI sites for cloning. We digested each fragment with XbaI and BamHI and inserted it into a eukaryotic expression vector, $\mathrm{CMV} /$ $\mathrm{R}^{14}\left(\mathrm{C}-\mathrm{E}_{37997}, \mathrm{C}-\mathrm{E}_{\mathrm{OPY}-1}, \mathrm{E}_{37997}\right.$ and $\left.\mathrm{E}_{\mathrm{OPY}-1}\right)$. The $\mathrm{CMV} / \mathrm{R}$ vector comprises the human cytomegalovirus (CMV) immediate early enhancer/promoter, a human $\mathrm{T}$ cell leukemia virus- $1 \mathrm{R}$ region containing a splicing donor, a CMV immediate early splicing acceptor and bovine growth hormone poly A signal.

Production of pseudotyped lentiviral vectors. We created lentiviral vectors expressing glycoproteins from 37997 and LR2006 OPY-1 CHIKV strains. The method for producing recombinant lentiviral vectors expressing a luciferase reporter gene has been previously described ${ }^{12,14}$. Briefly, we transfected 293T cells with 500 ng CHIKV envelope plasmid from either strain ( $\mathrm{E}_{37997}$ or $\left.\mathrm{E}_{\mathrm{OPY}-1}\right)$, $7 \mu \mathrm{g}$ of a transducing vector encoding a luciferase reporter gene under the control of a CMV promoter ( $\mathrm{pHR}$ 'CMV-luciferase plasmid) and $7 \mu \mathrm{g}$ of a packaging plasmid that expresses all HIV-1 structural proteins except envelope (pCMV $\Delta$ R8.2) (Supplementary Fig. 1a). Additional methods and the neutralization assay with CHIKV E-pseudotyped lentiviral vectors are described in the Supplementary Methods.

Buoyant density gradient sedimentation analysis and purification of viruslike particles. We transfected $293 \mathrm{~F}$ cells $\left(2.5 \times 10^{8}\right)$ (Invitrogen) with 293 fectin transfection reagent (Invitrogen) and $125 \mu \mathrm{g}$ of $\mathrm{C}^{-\mathrm{E}_{37997}}$ plasmid following the manufacturer's recommendations. Detailed methods for buoyant density gradient analysis and purification of VLPs have been previously described ${ }^{33}$ and are in the Supplementary Methods.

Cryoelectron microscopy and image analysis. We flash-froze Chikungunya VLPs on holey grids in liquid ethane and recorded images at $\times 47,000$ magnification with a CM300 field emission gun microscope with electron dose levels of approximately 20 electrons per $\AA^{2}$. We digitized all micrographs at $6.35 \mu \mathrm{m}$ per pixel using a Nikon scanner, and we boxed individual particle images using the program e2boxer in the EMAN2 package (National Center for Macromolecular Imaging $)^{34}$. We used the CTFIT program from the EMAN package ${ }^{35}$ to determine the contrast transfer function parameters and flip phases. We constructed an initial model in EMAN using assigned two-, three- and fivefold views and refined it in EMAN assuming icosahedral symmetry. The number of particles incorporated into the final reconstruction was 1,489 , giving a final resolution of $18 \AA$ on the basis of a 0.5 Fourier shell correlation threshold.

Immunizations and challenge of mice and monkeys. We mixed $19 \mu \mathrm{g}$ of VLPs (equivalent to approximately $10 \mu \mathrm{g}$ of E1-E2) in $60 \mu \mathrm{l}$ normal saline with $60 \mu \mathrm{l}$ of Ribi solution (Sigma Adjuvant system, Sigma-Aldrich) per mouse. We injected female 6- to 8-week-old BALB/c mice in the right and left quadriceps muscles with VLPs in normal saline or Ribi in $120 \mu \mathrm{l}$ total volume, two times at weeks 2 and 6. For DNA vaccination, we injected mice in the right and left quadriceps with a total of $15 \mu \mathrm{g}$ of purified plasmid C-E $\mathrm{E}_{37997}, \mathrm{E}_{37997}, \mathrm{C}-\mathrm{E}_{\mathrm{OPY}-1}$ or $\mathrm{E}_{\mathrm{OPY}-1}$ suspended in $100 \mu \mathrm{l}$ of normal saline three times at weeks 0,3 and 6 . Each group contained five mice. We collected sera and spleens $10 \mathrm{~d}$ after the last injection.

In monkey experiments, we injected 3- to 4-year-old rhesus macaques weighing 3-4 kg intramuscularly in the anterior quadriceps with either $20 \mu \mathrm{g}$ of VLPs in $1 \mathrm{ml}$ PBS (VLP group) or $1 \mathrm{~mL}$ PBS alone (control group) at weeks 0,4 and 24. Each group contained six monkeys. To measure antibody titers, we collected blood on days $-14,0,10,28,38,56,70,161$ and 178 . We challenged the monkeys ( $n=3$ per group, randomly selected from each group) with $1 \times 10^{10} \mathrm{PFU}$ of CHIKV (strain LR2006 OPY-1) 15 weeks after the final immunization by intravenous injection. We collected blood to measure viremia at $0,6,24,48,72,96$, 120 and $168 \mathrm{~h}$ and killed the monkeys $168 \mathrm{~h}$ after challenge. All animal experiments were reviewed and approved by the Animal Care and Use Committee, Vaccine Research Center, National Institute of Allergy and Infectious Diseases in accordance with the relevant federal guidelines and regulations.

Passive transfer of immunoglobulin and challenge in Ifnarl $^{-/-}$mice. We used a HiTrap Protein G HP column (GE Healthcare) and a Melon Gel IgG Purification Kit (Pierce) for purification of IgG from VLP-immunized or control monkeys. We dialyzed purified IgG three times against PBS. We administered $2 \mathrm{mg}$ of purified IgG (from approximately $200 \mu \mathrm{l}$ of serum) into each recipient Ifnarl ${ }^{-/-}$ mouse by tail vein injection $24 \mathrm{~h}$ before challenge. The challenge consisted of 30 PFU of CHIKV (strain LR2006 OPY-1), administered by intradermal injection.

Statistical analysis. All results are expressed as means \pm s.e.m. We analyzed the data with unpaired two-tailed $t$ tests (Prism 5).

Additional methods. Additional methods are described in the Supplementary Methods.

34. Tang, G. et al. EMAN2: an extensible image processing suite for electron microscopy. J. Struct. Biol. 157, 38-46 (2007).

35. Ludtke, S.J., Baldwin, P.R. \& Chiu, W. EMAN: semiautomated software for high-resolution single-particle reconstructions. J. Struct. Biol. 128, 82-97 (1999). 